Thorax (1966), 21, 28.

\title{
Rupture of the bronchus
}

\author{
R. L A R I Z A D E H
}

From the Department of Thoracic Surgery, Sully Hospital, Sully, Nr. Penarth, Glam.

Rupture of the bronchus has become more common in the last decade, mainly due to an increase in road traffic accidents. In spite of this increase it is still relatively rare, and most thoracic surgeons do not have the personal experience of treating more than two or three cases in their life-time.

In 1947 Kinsella and Johnsrud reviewed the world literature on intrathoracic rupture of the tracheo-bronchial tree and were able to collect 40 cases, none of which had been treated by primary repair of the lesion.

The first successful repair of a bronchial injury due to a penetrating wound was performed by Paul Sanger in 1945, but it was not until 1949 that Griffiths successfully repaired a bronchus which had completely ruptured eight months previously.

By 1959, in their review of the world literature, Hood and Sloan were able to collect 91 cases of tracheo-bronchial rupture. To this they added seven of their own. This increase over a period of 12 years reflects not only an increased incidence but also an increased awareness of the problem by the surgical profession.

\section{CASE REPORTS}

CASE 1 S. T., a boy aged 7 years, was injured by a heavy girdle which fell over the front of his chest. When freed he got up saying that he was going home, but he collapsed immediately afterwards.

On admission to Morriston Hospital on 13 June 1961 he was screaming, distressed, and deeply cyanosed. He also had gross subcutaneous emphysema of the whole body and was bleeding from the nose and mouth. Radiographs showed bilateral pneumothorax, pneumopericardium, and mediastinal emphysema together with a fracture of the fifth left rib. He was given oxygen, and pneumothorax needles (Martin's cannulae) were inserted into the right and left second intercostal spaces anteriorly, with resultant expansion of both lungs.

The cannulae became blocked on the third and fourth days respectively and had to be re-inserted. The cannula was removed from the left side on 22 June and from the right side on 27 June. Both lungs remained fully expanded. The patient continued to progress and was discharged home on $14 \mathrm{July}$, when the radiograph was normal and the only clinical abnormality was a slightly diminished air entry on the left side of the chest.

Two days later the boy was admitted to the local general hospital with abdominal pain and vomiting, and on examination was found to have follicular tonsillitis and partial collapse of the left lung. He was treated with antibiotics and physiotherapy without any improvement in the left lung, and was transferred back to Morriston Hospital on 11 August 1961. He was found to be acyanotic and had no dyspnoea. The left lung was completely collapsed. Bronchoscopy performed a few days later showed constriction of the left main bronchus just beyond the carina. This was dilated with gum elastic bougies, and the bronchogram showed a loculated air pocket due to a fistulous communication with the left main bronchus.

The boy was transferred to Sully Hospital two weeks later when a repeat bronchoscopy showed the carina to be normal, but about $1.5 \mathrm{~cm}$. beyond it the left main bronchus was stenosed to one-tenth of its normal size. This area was rigid and could not be dilated.

On 12 October 1961 a left thoracotomy was carried out (Mr. D. M. E. Thomas) ; the left lung was found to be completely atelectatic and the hilum was involved in a mass of fibrous tissue. After some dissection the pulmonary end of the left main bronchus was found and opened, and this released some mucopurulent material which was found to be sterile on culture. There was a gap of about $0.75 \mathrm{~cm}$. between this and the carinal end, which was also completely blocked. The two ends were cleaned and trimmed. An end-to-end anastomosis was performed, using interrupted sutures of fine silk. Prior to anastomosis the lung was inflated and was found to expand with surprising ease.

The post-operative period was uneventful ; the lung expanded immediately and remained fully expanded. In the immediate post-operative period postural 0 drainage of the operated lung was considered to be 0 essential. The patient was discharged home on $26 \stackrel{\varnothing}{\complement}$ November 1961, symptomless and with no abnormal of physical signs in the chest. The left lung was fully expanded.

He was seen in the out-patient department at $\stackrel{D}{\mathbb{D}}$ frequent intervals and was readmitted to hospital in $\frac{?}{\mathbb{D}}$ March 1962 because of diminished air entry on the $\unrhd$ left side of the chest with radiological findings suggestive of obstructive emphysema of the left lung. 
Bronchoscopy showed the presence of granulation tissue at the site of the anastomosis, diminishing the calibre to half the normal size. This was dilated with ease. Identical presentation and findings were repeated in July 1962 and February 1963. These were treated as above with a satisfactory result. The patient was last readmitted on 1 April 1965 when bronchoscopy showed no granulation tissue and the lumen was only slightly smaller than normal, as confirmed by bronchogram.

CASE 2 A. L., a boy aged 6 years, was injured by a pole falling across his left chest, and he was admitted to the local general hospital on 13 November 1964. On admission he was found to be slightly dyspnoeic but was not shocked or cyanotic. He had a tension pneumothorax but no subcutaneous emphysema. He was treated by pleural drainage and under-water suction using a Martin's cannula. When the cannula was removed on 16 November the lung was found to be fully expanded. However, the patient's general condition was not satisfactory due to general malaise and pyrexia, and on 19 November he developed atelectasis of the left lung. He was transferred to Morriston Hospital and was pale with a pyrexia of $100 \cdot 4^{\circ} \mathrm{F}$. and a pulse rate of 120 per minute. Examination of the chest showed the presence of a pleuro-pericardial friction rub along the left sternal edge with absent air entry over the left lung, except for a small area over the left upper lobe where bronchial breath sounds were present. The patient was treated with antibiotics and physiotherapy, with resultant full expansion of the lung by 21 November. However, by 24 November the left lung had again become atelectatic after having been expanded for over 48 hours.

On 25 November bronchoscopy showed the carina to be normal. The left upper lobe orifice was seen, but, due to welling up of blood from the left lower lobe region, a proper assessment was impossible. Over the next few days there was some improvement in the patient's general condition and a repeat bronchoscopy on 30 November showed a complete block of the left main bronchus just proximal to the left upper lobe orifice.

On 4 December he was transferred to Sully Hospital, where he was found to be pale and slightly dyspnoeic with signs of collapse of the left lung. A repeat bronchoscopy confirmed the above findings.

On 4 December a left thoracotomy was carried out (Mr. Cyril Evans). The left lung was found to be completely atelectatic and the left main bronchus was surrounded by a mass of fibrous tissue. The main bronchus was dissected free and was found to be severed just above the left upper lobe orifice. The gap between the two ends was filled by tough fibrous tissue. The distal bronchus was opened and found to be full of light yellow mucus which was sterile on culture. The two bronchial stumps were trimmed and an end-to-end anastomosis was carried out using 10 interrupted sutures of fine silk. After this the lung expanded satisfactorily, and the patient had a smooth post-operative period. Again special emphasis was placed on postural drainage during the immediate post-operative period. The patient was discharged home in January 1965 and when last seen as an out-patient he was symptomless and the radiological findings were satisfactory.

\section{DISCUSSION}

Traumatic tears of the major bronchial tree may be associated with a trivial injury or accompanied by other serious, and at times lethal, problems. These injuries are usually the result of crush injuries to the chest, most commonly due to road accidents, but occasionally they arise from direct blows to the chest. At the time of the accident the fixed, more rigid bronchus with its more resistant wall ruptures, whereas the soft mobile structures of the hilum and mediastinum usually escape injury.

The exact mechanism of the bronchial rupture in closed chest injury is incompletely understood, but a number of theories have been put forward to explain the mechanism:

(1) A simple shearing force acting on the lung and bronchi (Sale, 1954).

(2) Schönberg's (1912) theory that if a chest injury occurs when the glottis is closed (its usual position in anticipation of imminent accident) a very high intraluminal pressure is created in the tracheo-bronchial tree, far in excess of the intrathoracic pressure, and this is responsible for the rupture.

(3) The theory put forward by Richards and Cohn (1955) that in closed chest injuries there must be a sudden increase in intrabronchial pressure against a closed glottis. This results in a regional strain or shearing force on the bronchial tree, thereby producing the rupture.

Rupture of the bronchus usually occurs in the main bronchus at or near the carina. Although rupture of all the individual bronchi has been recorded, the commonest site of rupture is about 1 to $2 \mathrm{~cm}$. from the carina. This is a relatively fixed portion of the bronchial tree. The two sides are affected equally, and contusion of lung parenchyma is not a common association of this injury.

In a study of 80 cases in which the information was available, Hood and Sloan (1959) found that in 42 patients no other injury was present. Of the remaining 38, 26 had fractures of the thoracic cage, five had fractures of the pelvis, four had fractures of long bones, three had head injuries, two had spinal cord injuries, and two cases had rupture of the oesophagus. 
PATHOLOGY The pathological changes occurring in the ruptured bronchus and the affected lung in patients not treated by immediate surgical repair depend on whether the rupture is incomplete or complete.

In incomplete rupture the bronchial tree is partially blocked by granulation tissue developing at the site of the rupture. This is later replaced by fibrous tissue, and eventually fibrous stricture of the bronchus takes place. These cases are invariably infected. The lung first becomes bronchiectatic and later atelectatic and fibrotic. When this state of infection and destruction has occurred, pulmonary resection is the only possible treatment that can be given, since attempts at dilatation are nearly always unsatisfactory.

When complete bronchial rupture occurs, the two bronchial stumps heal by granulation and completely close the openings. In the meantime the mucous glands continue to secrete mucus which distends the distal bronchial tree. Infection is uncommon in complete rupture, and in its absence irreversible bronchiectasis and parenchymal lung damage are not usually seen. However, occasionally some intrapulmonary fibrosis may occur. These cases of complete rupture offer the possibility of late reconstruction of the bronchus with successful aeration of the distal lung.

There is no obvious explanation why some lungs will expand after a long period of collapse whereas others will not. In lungs that do not expand, the atelectatic lung shows peribronchial and perivascular fibrosis and large amounts of collagen, with a moderate amount of plasma cell and lymphocytic infiltration.

Clinical PICTURE The patient may have an isolated injury of the bronchial tree or it may be accompanied by severe local or distal injuries. If shock is present it is usually due to loss of blood from other injuries or to cardiac compression from mediastinal air. In airway injuries the respiration is shallow and rapid and often accompanied by cyanosis. This respiratory embarrassment is mainly due to blood in the tracheobronchial tree, lack of aeration of the affected lung, and interference with proper aeration of the unaffected lung. Subcutaneous emphysema is usually present, involving the neck and the upper part of the chest at the onset but later becoming more widespread. This is often, but not invariably, accompanied by mediastinal emphysema, in which case a xiphisternal click and mediastinal crunch associated with the heart beat can be heard.
In intrathoracic rupture of the bronchus a pneumothorax is nearly always present. This may be of simple or tension variety. However, pneumothorax, tension pneumothorax or subcutaneous emphysema may be absent.

Chest radiographs may confirm the presence of any of the above conditions, but the picture is often confused by emphysema, blood in the lung or pleura, and changes brought about by possible injuries to the thoracic cage. With pleural intubation, expansion of the lung usually occurs, but this is eventually followed by atelectasis which is occasionally misdiagnosed as pleural effusion.

A large air leak through the intercostal tube is suggestive of bronchial rupture, and when the patient's condition worsens on application of high suction to the intercostal tube bronchial rupture must be suspected. In this event most of the inspired air is sucked out through the open bronchus and so prevented from ventilating the unaffected lung.

Bronchoscopy is indicated whenever (a) a patient after exposure to trauma complains of blood-stained sputum and cough accompanied by distressed respiration; (b) a pneumothorax is present which does not respond to pleural drainage ; and $(c)$ a very large air leak is present on pleural suction.

The bronchial tear may not be seen because of an excessive amount of blood around the region of the ruptured bronchus. In patients in whom the bronchoscopy is carried out some days after the injury, excessive granulation and bronchial stenosis may be found.

\section{CONCLUSION}

Our two cases help to emphasize a few important aspects of rupture of the bronchus:

1. The diagnosis of ruptured bronchus is not always straightforward, and, unless it is constantly considered in cases of collapsed lung and haemoptysis following any chest injury, it may be missed or delayed.

2 . In children, rupture of a bronchus can occur without injury to the thoracic cage.

3. It is possible for the lung distal to the ruptured bronchus to expand fully in the early days after injury, and this possibility must always be kept in mind. This may be explained by the assumption that it is possible for the major part of the mucosal layer lining the damaged bronchus still to be intact, and it is only when healing occurs and granulation tissue starts to form that the bronchial lumen becomes occluded. 
4. Post-operatively, close follow-up is essential, and frequent bronchoscopy and dilatation may be necessary in the early days before a final satisfactory result is achieved.

\section{SUMMARY}

Two cases of ruptured bronchus are discussed with a brief review of the literature. Essential points in diagnosis and treatment are discussed, and in conclusion important points are emphasized.

I would like to thank Mr. D. M. E. Thomas and Mr. C. Evans for allowing me to publish these two cases and also for their invaluable assistance. I am also very grateful to Mr. H. R. S. Harley for his helpful criticism of the paper.

My thanks are given also to Miss P. Morse, of the Department of Surgery, Sully Hospital, and to Mr. F. Midgely, of the Department of Medical Photography.

\section{REFERENCES}

Griffiths, J. L. (1949). Fracture of the bronchus. Thorax, 4, 105. Hood, R. M., and Sloan, H. E. (1959). Injuries of the trachea and major bronchi. J. thorac. cardiovasc. Surg., 38, 458.

Kinsella, T. J., and Johnsrud, L. W. (1947). Traumatic rupture of the bronchus. J. thorac. Surg., 16, 571.

Richards, V., and Cohn, R. B.(1955). Rupture of the thoracic trachea and major bronchi following closed injury to the chest. Amer. $J$. Surg., 90, 253.

Sale, T. A. (1954). Fracture of the bronchus. Brit. J. Surg., 41, 625.

Sanger, P. W. (1948). Evacuation hospital experiences with war wounds and injuries of the chest. Ann. Surg., 122, 147.

Schönberg, S. (1912). Berl. klin. Wschr., 49, 2218. 